\title{
SoftCuts: A Soft Edge Smoothness Prior for Color Image Super-Resolution
}

\author{
Shengyang Dai, Student Member, IEEE, Mei Han, Wei Xu, Ying Wu, Senior Member, IEEE, Yihong Gong, and \\ Aggelos K. Katsaggelos, Fellow, IEEE
}

\begin{abstract}
Designing effective image priors is of great interest to image super-resolution (SR), which is a severely under-determined problem. An edge smoothness prior is favored since it is able to suppress the jagged edge artifact effectively. However, for soft image edges with gradual intensity transitions, it is generally difficult to obtain analytical forms for evaluating their smoothness. This paper characterizes soft edge smoothness based on a novel SoftCuts metric by generalizing the Geocuts method [1]. The proposed soft edge smoothness measure can approximate the average length of all level lines in an intensity image. Thus, the total length of all level lines can be minimized effectively by integrating this new form of prior. In addition, this paper presents a novel combination of this soft edge smoothness prior and the alpha matting technique for color image SR, by adaptively normalizing image edges according to their $\alpha$-channel description. This leads to the adaptive $S$ oftCuts algorithm, which represents a unified treatment of edges with different contrasts and scales. Experimental results are presented which demonstrate the effectiveness of the proposed method.
\end{abstract}

Index Terms- $\alpha$-channel description, edge smoothness, SoftCuts, super-resolution (SR).

\section{INTRODUCTION}

I MAGE super-resolution (SR) [2] is the problem of recovering high-resolution (HR) images from low-resolution (LR) inputs. This problem is of great practical interest, especially to video communication, object recognition, HDTV, image compression, etc. This is a very challenging task, especially when only one single LR input image is available (see, for example, [3]), as is very often the case. This paper mainly focuses on this case.

In theory, the generation process of LR images can be characterized by smoothing and down-sampling the HR scenes with low-quality image sensors. Recovering the original HR image

Manuscript received June 14, 2008; revised November 10, 2008. Current version published April 10, 2009. This work was supported in part by National Science Foundation Grant IIS-0347877. The associate editor coordinating the review of this manuscript and approving it for publication was Dr. Pier Luigi Dragotti.

S. Dai, Y. Wu, and A. K. Katsaggelos are with the Department of Electrical Engineering and Computer Science, Northwestern University, Evanston, IL 60208 USA (e-mail: s-dai@northwestern.edu; yingwu@eecs.northwestern. edu; aggk@eecs.northwestern.edu).

M. Han is with Google, Inc., Mountain View, CA 94043 USA (e-mail: meihan@google.com).

W. Xu and Y. Gong are with NEC Laboratories America, Inc., Cupertino, CA 95014 USA (e-mail: xw@sv.nec-labs.com; ygong@sv.nec-labs.com).

Color versions of one or more of the figures in this paper are available online at http://ieeexplore.ieee.org.

Digital Object Identifier 10.1109/TIP.2009.2012908 from LR inputs is an inverse process. One intuitive approach to address this inverse problem is to minimize the reconstruction error, which is defined as the difference between the observation and the result obtained by putting the recovered HR image through the same generation process. In other words, the result which can produce the closed LR image to the observed one is preferred. Such a reconstruction error can be efficiently optimized by the back-projection method [4] in an iterative way. However, researchers have found that the SR problem is essentially under-determined [5], [6]. Given the LR input or inputs, there might be multiple solutions that can minimize the reconstruction error. Thus, by simply minimizing the reconstruction error, the result may converge to an unsatisfying solution. To overcome this difficulty, it is necessary to regularize the under-determined inverse problem by incorporating effective image priors.

Simple interpolation-based methods, such as bilinear or bicubic interpolation tend to produce HR images with jagged edges, which is also a common artifacts for many SR algorithms. This observation motivates us to design an edge smoothness prior that favors an HR image with smooth edges. In addition, this prior is also consistent with human perception. However, designing and incorporating an effective edge smoothness prior is a very challenging task due to the following two main difficulties in practice.

First, it is very difficult to quantitatively characterize the smoothness of an edge, especially for edges in natural images. In most real situations, an image edge exhibits a gradual intensity transition, which is in general much more complex than a simple geometric curve. In this paper, we refer to such an edge as a soft edge [see Fig. 1(b) for an example], as opposed to a hard edge, as shown in Fig. 1(a). Working with soft edges poses an extra difficulty in measuring and quantifying the edge smoothness in an analytical form.

Second, color image edges are determined by the information from all three color channels simultaneously. Besides, edges in natural images exhibit a large variation with different contrasts and scales. How to explore the 3-D color information and treat those various edges in a unified way is of great importance to color image SR.

This paper is mainly focused on addressing the above two issues. The main contributions are as follows.

1. To measure and quantify the edge smoothness, we introduce the Geocuts method [1], which can approximate the Euclidean length of a hard edge with a cut metric on the image grid. More importantly, in order to handle soft edges, we propose an extension, i.e., SoftCuts method based on 


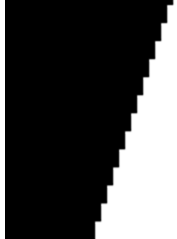

(a)

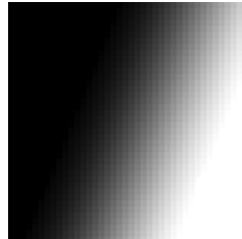

(b)

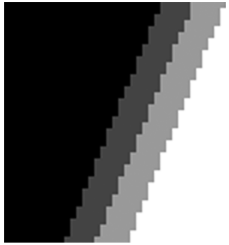

(c)
Fig. 1. Examples of (a) hard edge, (b) soft edge, and (c) level lines (boundaries between different intensities) for (b) with quantization step size equal to 64 on image intensities.

a soft edge cut metric, which can measure the smoothness of soft edges in an intensity image. This extension is significant because it leads to a new analytical form for the soft edge smoothness prior. The new smoothness measure is used to regularize the objective function of the SR task, and produce impressive results. This metric also has a nice geometric property, since it approximates the average length of all level lines in the intensity image, where a level line represents the boundary between pixels with intensities smaller and larger than a given value [an example is shown in Fig. 1(c)].

2. To handle various edges in color images, a novel image representation scheme is proposed based on the alpha matting technique from the computer graphics literature. We transform the problem of color edge SR to a combination of alpha matting decomposition and $\alpha$-channel SR. This makes possible the application of the soft edge smoothness prior to natural color images.

3. To process the entire image in a unified way, an adaptive strategy is further proposed to utilize the SoftCuts for all image edges simultaneously by using an $\alpha$-channel image description obtained with a multiple layer image matting decomposition method [7]. Due to this decomposition, the SoftCuts regularization term for different edges is normalized, such that the smoothness measure of all level lines in the $\alpha$ channel has the same weight.

The proposed SR algorithm has the following benefits: 1) Due to the geometry property of the proposed SoftCuts measure, the length of all image level lines can be minimized simultaneously for the SR task. Thus, results with smooth edges can be obtained. In the mean time, the edge-preserving property of the proposed prior term can also make the resulting edges have sharp transitions. 2) All three color channels are utilized simultaneously with the $\alpha$-channel SR scheme, and the adaptive strategy provides a unified treatment of edges with different contrasts and scales.

In this paper, the related work is summarized in Section II. The SoftCuts method is proposed in Section III, and the geometric explanation is presented. This SoftCuts metric can be used as a regularization term in the SR problem to favor soft smooth edges. To make use of this term adaptively, we first introduce the $\alpha$-channel edge/image description method in Section IV. Based on that, an adaptation scheme is derived in Section V, which applies the SoftCuts regularization term with the same weight on different edge transitions. Experiments are shown in Sections VI and VII concludes this paper.

\section{RELATED WORK}

Due to the under-determined nature of the SR problem, an image prior needs to be utilized to regularize the restoration process. Extensive work has been done on SR given multiple LR images [2], [4], [8], [9]. The prior term (or the image regularization term) in these works could also be applied to the single image SR problem directly. So in this section, we will also review the methods used when multiple LR images are available. In general, having multiple LR input images imposes more constraints on the likelihood term (or the image fidelity term). Thus, it is argued that the use of an effective image prior is even more critical for single image SR.

Existing works on the use of image priors for SR tasks can be placed into two categories: priors from image modeling and priors from learning.

In the first case, based on the observation that neighboring pixels tend to have the same color, the image smoothness prior is widely explored, such as in various filtering or interpolation algorithms (e.g., bilinear and bicubic interpolation). Such methods usually produce blurry images, since the image edges are also smoothed. Edge-preserving regularization terms [9]-[11] are designed to address the over-smoothness problem at image boundaries. In [9], the bilateral prior is used for single channel images. The high order neighborhood is also investigated in this work, but from a different perspective from ours. This work is extended to color images [12] by optimizing a multiterm cost function, which is a combination of regularization terms on illuminance and chrominance. In [10], the sparse derivative prior is applied, which can preserve sharp edges by modeling the image derivative with a heavy-tailed distribution, instead of traditional Gaussian distribution. In [11], a TV prior is used along with a variational approximation for obtaining the solution. Another popular approach is using edge directed interpolation [13]-[15], which is trying to infer sub-pixel edges positions, to further prevent cross-edge interpolation. Locating the edge position with high precision is of great importance for those methods. Another common observable artifact of SR algorithms is the jagged edge effect. It is reasonable to assume an edge smoothness prior without any other prior knowledge on the image. This prior is also consistent with human perception, which seems to also favor smooth curves in natural images. Based on this assumption, a lot of algorithms have been proposed to obtain smooth edges. Various techniques are investigated in the literature to obtain smooth image boundaries, such as level-set [16], multiscale tensor voting [17], and snake-based vectorization [18] techniques.

Instead of modeling the image prior explicitly, many researchers try to learn the prior from natural images, especially by learning from image exemplars directly. One common way is to infer the missing high frequency information for the HR images for each position based on the middle frequency information. This approach is adopted in [19] for the image restoration problem. The Markov random fields model [20]-[22] is usually utilized to enforce the spatial consistency. Extension of this research direction includes video SR [23] and learning from domain-specific exemplars [24]. In such methods, two key problems usually need to be addressed. The 
first is how to search HR candidate patches efficiently. Locality sensitive hashing [25] and KD-tree [24] are applied to speed up the searching. The second is how to solve the optimization problem efficiently. Belief propagation can be used, and the image primal sketch [26] method can simplify the problem to a chain structure. Other learning algorithms can also be used to infer the high-frequency information, such as locally linear embedding [27].

This work is an extension of the authors' previous work on $\alpha$-channel edge SR [28]. This paper presents a more detailed and comprehensive treatment of the topic, and introduces the local adaptive scheme under the framework of $\alpha$-channel SR, in order to enable a unified treatment of the entire image.

\section{Soft Edge SMOOTHNESS PRIOR}

In this section, we first review the Geocuts method which is designed for image segmentation tasks, and then present our work of the SoftCuts method for measuring the soft edge smoothness.

\section{A. Geocuts}

Our work is partially motivated by the Geocuts method [1], which is briefly summarized in this section.

The basic idea of Geocuts is to use a cut metric to approximate the Euclidean length of a curve. The cut metric is defined on the image grid graph, with edges connecting pixels in a large neighborhood system, and proper edge weights. Since such a cut metric has an analytic form, the smooth hard edges can be obtained by minimizing this metric.

More formally, the weighted grid-graph $\mathcal{G}=\langle\mathcal{V}, \mathcal{E}\rangle$ is designed in the following way. $\mathcal{V}$ is the set of all discretized image pixel positions. The edge set $\mathcal{E}$ is defined according to the neighborhood system represented by a set of vectors $\left\{e_{k} \mid 1 \leq\right.$ $\left.k \leq n_{\mathcal{G}}\right\}$, where $n_{\mathcal{G}}$ is the neighborhood order, and the $e_{k}$ 's are chosen as the relative position (taking integer values as its components, and the unit is the grid interval) of the nearest $n_{\mathcal{G}}$ neighbors with different directions. They are ordered by their corresponding angle $\phi_{k}$ w.r.t. the $+x$ axis, such that $0 \leq \phi_{1}<$ $\phi_{2}<\cdots<\phi_{n_{\mathcal{G}}}<\pi$. For example, when $n_{\mathcal{G}}=4$, we have $e_{1}=(1,0), e_{2}=(1,1), e_{3}=(0,1)$, and $e_{4}=(-1,1)$. The set of pixel pairs with relative position $e_{k}$ is denoted by $\mathcal{N}_{k}$, assuming that $\mathcal{N}=\cup_{k=1}^{n_{\mathcal{G}}} \mathcal{N}_{k}$. All pixel pairs with relative position inside the set $\mathcal{N}$ are connected by edges. Fig. 2 shows some example neighborhood systems. After defining the edges, the edge weights corresponding to $\mathcal{N}_{k}$ are defined as

$$
w_{k}=\frac{\delta^{2} \Delta \phi_{k}}{2\left|e_{k}\right|}
$$

where $\Delta \phi_{k}=\phi_{k+1}-\phi_{k}\left(\right.$ set $\left.\phi_{n_{\mathcal{G}}+1}=\pi\right)$, and $\delta$ is the size of the grid interval.

Given the weighted grid-graph $\mathcal{G}$ and a curve $C$ in $\mathbb{R}^{2}$ overlayed on $\mathcal{G}$, we denote by $\mathcal{E}_{C}$ the set of edges intersecting this curve. The cut metric of $C$ is defined as

$$
|C|_{\mathcal{G}}=\sum_{e \in \mathcal{E}_{C}} w_{e}
$$


Fig. 2. Neighborhood systems for $n_{\mathcal{G}}=2,4$ (left) and $n_{\mathcal{G}}=12$ (right, only the neighbors on the upper plane are shown).

where $w_{e}$ is the weight for edge $e$. In other words, $|C|_{\mathcal{G}}$ is the summation of the weights of all edges intersecting $C$. Denoting by $|C|_{\mathcal{E}}$ the Euclidean length of curve $C$, the following theorem is derived in [1].

Theorem 1: If $C$ is a continuously differentiable regular curve in $\mathbb{R}^{2}$ intersecting each straight line a finite number of times then

$$
|C|_{\mathcal{G}} \rightarrow|C|_{\mathcal{E}}
$$

as $\delta, \sup _{k}\left|\Delta \phi_{k}\right|$, and $\sup _{k}\left|e_{k}\right|$ go to zero [1].

Theorem 1 means that the length of a curve can be approximated by its cut metric on a weighted image grid graph. It is derived from the Cauchy-Crofton formula in integral geometry, which relates the Euclidean length of a curve with the expectation of the number of intersections with a random line. This expectation is further approximated by the cut metric defined in (2) with the image grid graph and appropriately chosen edge weights. Roughly speaking, the approximation error converges to zero as the grid size goes to zero and a larger neighborhood is considered. This method can be generalized to 3-D and arbitrary Riemannian metric. The global minimum of the cut metric can be obtained in a close-to-linear time by the graph cuts method [29], [30]. As its name suggests, Geocuts reveals the underlining relationship between two well-known segmentation algorithms, i.e., Geodesic active contours and graph cuts. Geocuts also provides a principled way to choose the edge weights for using higher order neighborhoods.

By integrating the cut metric into an objective function, the hard edge smoothness prior can be added. Curves with smaller Euclidean length are preferred by minimizing such an objective function, thus smooth curves are obtained.

\section{B. Softcuts for Smoothness Measure of Soft Edges}

Now, we present the proposed SoftCuts method as a generalization of the Geocuts method.

A cut metric can be defined on any set of disjoint closed curves $C$, or equivalently, a binary valued characteristic function $\chi^{C}$ on $\mathbb{R}^{2}$ which equals 1 inside $C$, and 0 , otherwise. Geocuts is only applicable to a binary valued function $\chi^{C}$ on the image plane. To handle soft edges, which are gradual transitions in an intensity image, we first rewrite the definition of the cut metric in (2) w.r.t. curve $C$ (or equivalently, function $\chi^{C}$ ) as follows:

$$
\begin{aligned}
|C|_{\mathcal{G}} & =\left|\chi^{C}\right|_{\mathcal{G}} \\
& =\sum_{1 \leq k \leq n_{\mathcal{G}}}\left(w_{k} \sum_{(p, q) \in \mathcal{N}_{k}}\left|\chi_{p}^{C}-\chi_{q}^{C}\right|\right)
\end{aligned}
$$




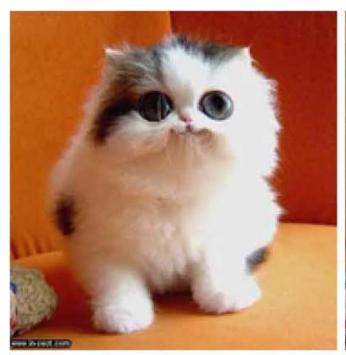

(a)

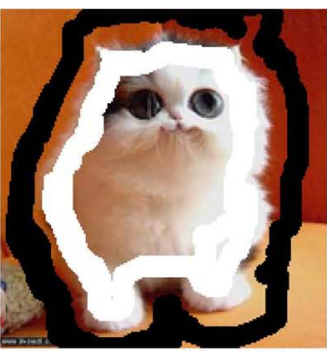

(b)

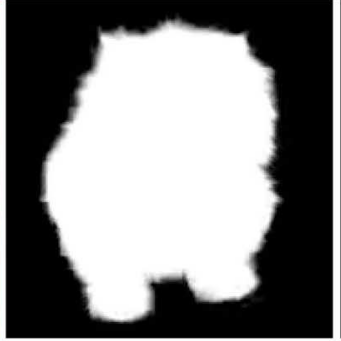

(c)

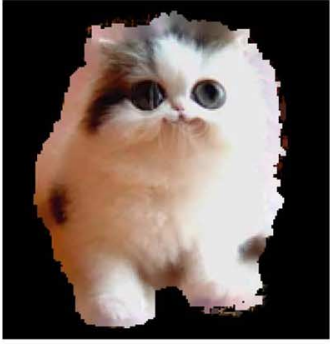

(d)

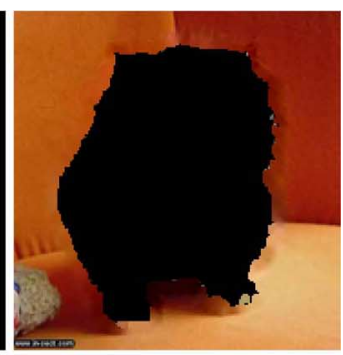

(e)

Fig. 3. Example of the alpha matting technique. (a) Input color image I. (b) Human interaction specifying pure foreground and background pixels with black and white strokes. (c) Extracted $\alpha$ channel (white represents 1, and black represents 0). (d) Foreground patch F. (e) Background patch B.

where $p$ and $q$ are pixel positions on the grid $\mathcal{G}$; they are used as subscripts to indicate the function value at that pixel for simplicity throughout this paper. We simplify the above equation as

$$
|C|_{\mathcal{G}}=\sum_{(p, q) \in \mathcal{N}} w_{p q}\left|\chi_{p}^{C}-\chi_{q}^{C}\right|
$$

where $w_{p q}$ is the weight for the neighboring pixel pair $(p, q)$, which is determined by the relative position of $p$ and $q$. In other words, for $(p, q) \in \mathcal{N}_{k}, w_{p q}=w_{k}$. Although (5) is equivalent to (2), the former is easier to be generalized to a real valued function $S$ on $\mathbb{R}^{2}$. We define the soft cut metric for $S$ w.r.t. the grid-graph $\mathcal{G}$ as follows:

$$
|S|_{\mathcal{G}}=\sum_{(p, q) \in \mathcal{N}} w_{p q}\left|S_{p}-S_{q}\right|
$$

Equation (6) takes the same form as (5), with the only difference that continuous values are allowed for $S$ in (6), instead of binary value for $\chi^{C}$ in (5).

By uniformly quantizing the function values with step $1 / n, S$ can be approximately by $S^{d}$, which takes values from $\{0,1 / n, 2 / n, \ldots, 1\}$. The soft cut metric of $S^{d}$ can be similarly defined by (6), by replacing $S$ with $S^{d}$. Moreover, $S^{d}$ can be equivalently described by a set of level lines $l_{1}, l_{2}, \ldots, l_{n}$, where $l_{i}$ is the boundary between regions with $S^{d}$ values that $S^{d}<i / n$ and $S^{d} \geq i / n$ in $\mathbb{R}^{2}$. From Theorem 1, we know that the length of $l_{i}$ can be approximated by its cut metric $\left|l_{i}\right|_{\mathcal{G}}$. Based on this, we have the following theorem (the proof is given in Appendix A).

Theorem 2: Assume that $S$ is a continuous differentiable regular function on $\mathbb{R}^{2}$, which ranges in $[0,1]$, and $S^{d}$ discretizes $S$ with step $1 / n$. Then the average length of all level lines of $S^{d}$ w.r.t. $1 / n$ can be approximated by the soft cut metric of $S^{d}$, i.e.,

$$
\left|S^{d}\right|_{\mathcal{G}} \rightarrow \frac{1}{n} \sum_{1 \leq i \leq n}\left|l_{i}\right|_{\mathcal{E}}
$$

under the same conditions of Theorem 1.

Theorem 2 generalizes Theorem 1 to soft edges from hard boundaries. It implies that by minimizing the soft cut metric in (6), the sum of lengths of discrete level lines can be minimized. So adding this metric as a regularization term can help us obtain results with smaller length of image level lines. Thus, the soft smoothness prior can be easily incorporated into the SR objective function.
There are several related works in the literature. The level-set method [16] and multiple-scale tensor voting [17] has been used to incorporate the edge smoothness prior. Image gradient on a large neighborhood is also used in [9] as a regularization term. Compared with these existing works, the benefit of the proposed SoftCuts method is that we have an explicit analytic term to characterize the edge smoothness, with a specific geometric explanation. When $n_{\mathcal{G}}=2$, (6) becomes an approximation to the total variation (TV) regularization term [31]-[33], which is very powerful in edge-preserving image reconstruction.

\section{IV. $\alpha$ Channel Image Decomposition}

For a color image I, matting is the technique that separates its foreground objects from the background. As the pixels on the object boundary tend to be mixtures of both foreground and background color components, the separation needs to be done softly. A color image can be treated as a linear combination of the foreground and background images. Specifically, for each pixel $p$, we have

$$
\mathbf{I}_{p}=\alpha_{p} \mathbf{F}_{p}+\left(1-\alpha_{p}\right) \mathbf{B}_{p}
$$

where $\mathbf{I}_{p}, \mathbf{F}_{p}$, and $\mathbf{B}_{p}$ are the pixel color vectors of the input, foreground, and background images, respectively, and $\alpha_{p} \in$ $[0,1]$ determines the weights of the convex combination of the foreground and the background at position $p$. Alpha matting tries to recover $\mathbf{F}, \mathbf{B}$, and $\alpha$ simultaneously for each pixel, given an input color image I. An example is shown in Fig. 3.

For a color image edge, if we consider the two sides of this edge one as the foreground layer $\mathbf{F}$ and the other as the background layer $\mathbf{B}$, then the region close to this edge can also be decomposed by alpha matting. Once having the decomposition, instead of using the original pixel colors, we use $\alpha$ to represent this edge, and refer to it as the $\alpha$-channel edge description.

The idea of using the $\alpha$-channel is attractive due to its special properties as described next. First of all, the $\alpha$-channel provides a normalization of the edge, that is, they transition from 0 to 1 between the two sides of the edge, instead of a large range of possible values in the color channels. More importantly, the $\alpha$-channel actually preserves the edge information in a single channel as we will see in Section V-C. So, it can be considered as a normalized version of the image edge. In addition, using the $\alpha$-channel can explore the relationship among three color channels and naturally combine all color information from three channels. In fact, color channels are closely related 
to each other. One example is the linear color model [34], which means that the pixels colors in a local image patch tend to form a line in the 3-D color space. Such information is totally ignored by methods that process three color channels separately. The $\alpha$-channel is extracted in a way such that all color information is taken into account simultaneously. Thus, the color channel relationship information is implicitly integrated.

The idea of using the $\alpha$-channel has been successfully applied to image deblurring [35]-[37]. Matting is also used in [17] to extract sub-pixel location of the curve for SR. A two color image prior, which in essence very similar to the matting decomposition, is used in [38] for demosaicing.

Given an input image $\mathbf{I}$, solving for $\alpha, \mathbf{F}$, and $\mathbf{B}$ simultaneously is obviously an under-determined problem. Image priors are needed to regularize the problem. One commonly used prior is the image smoothness prior, which assumes local color smoothness for both of the foreground and the background. Fig. 3 shows an example of a matting solution with this prior, where we can clearly see that $\mathbf{F}$ and $\mathbf{B}$ are locally smooth along the matting boundary, and $\alpha$ retains most of the edge information. This smoothness prior is extended to the local linear color model in [34]. Based on this assumption, a closed-form solution is derived. User interaction can be considered as another kind of prior, where some pure foreground/background pixels are indicated manually by providing a trimap or using a brush tool [Fig. 3(b)] [39].

Traditionally, the alpha matting technique decomposes an input image into two components, i.e., a foreground layer and a background layer. Very recently, the spectral matting technique [7] was proposed to generalize this concept by decomposing a color image into a linear combination of multiple image layers as follows:

$$
\mathbf{I}=\sum_{i=1}^{n} \alpha_{i} \mathbf{L}_{i}
$$

where $\mathbf{L}_{i}$ is the $i$-th image layer, $\alpha_{i}$ is the corresponding combination weight, satisfying $\sum_{i} \alpha_{i}=1$ for each pixel, and $n$ is the total number of layers. This method not only enables multiple layer image representation, but also leads to a fully automatic and unsupervised solution for $\alpha_{i}$ by the spectral clustering technique. Thus, human interaction that specifies partial foreground or background pixels is not necessary anymore. An example is shown in Fig. 4. Each layer roughly corresponds to a homogeneous image region, and the edge information is captured by at least one of those $\alpha$ channels.

The definition of $\alpha$-channel edge description can be easily extended to an $\alpha$-channel image description as $\mathbf{a}=\left(\alpha_{1}, \alpha_{2}, \ldots, \alpha_{n}\right)$, given the multiple-layered image decomposition. Similar to the $\alpha$-channel edge description, image edges in the $\alpha$-channel image description are also normalized in the range $[0,1]$ over one single channel. Pixels along the border of two neighboring image layers have two nonzeros components in their $\alpha$-channel image description, and these two values should sum up to 1 for each pixel. The $\alpha$-channel edge description and the $\alpha$-channel image description contain the same edge transition information for those pixels. Thus, all the properties of the $\alpha$-channel edge description can be



Fig. 4. Multiple layer image representation. The top left image is the input, the other five images represent the $\alpha$ value for the five layers.

directly applied to the $\alpha$-channel image description scheme in those regions. Although this property is not satisfied for pixels corresponding to more than two layers, such pixels only represent a very small percentage of the total number of pixels [7]. Moreover, the $\alpha$-channel image description integrates the information of all image edges simultaneously, instead of one single edge for the $\alpha$-channel edge description. This property makes the $\alpha$-channel image description more suitable since it enables a unified image processing solution, as shown later in Section V-C.

\section{SOFTCUTS FOR COLOR IMAGE SR}

In this section, we first apply the SoftCuts method directly to single channel image SR. Then we use the SoftCuts on $\alpha$ channel to super-resolve color image edges. Finally, the adaptive SoftCuts method is presented to process the entire color image in a unified framework.

\section{A. Single Channel SR by Softcuts}

Theoretically, the generation process of an LR image can be modeled by the combination of various blurs, such as atmospheric blur, motion blur, camera blur, out-of-focus blur, and down-sampling. By combining the various blurs into a single filter $G$ for the entire image, the generation process of a single channel LR image can be formulated as follows:

$$
I^{l}=\left(I^{h} * G\right) \downarrow+n
$$

where $I^{h}$ and $I^{l}$ represent the HR and LR single channel images, respectively, $G$ the impulse response of the blurring filter, $*$ the convolution operator, $\downarrow$ the down-sampling operator, and $n$ the additive noise.

We propose the SoftCuts method by utilizing the soft cut metric for single channel SR as the regularization term. The objective function is now defined as follows:

$$
I^{h}=\arg \min _{I}\left(\left\|I^{l}-(I * G) \downarrow\right\|_{2}^{2}+\lambda|I|_{\mathcal{G}}\right)
$$

where the first term on the right side represents the fidelity to the data, the second term represents our prior knowledge about the original image defined by (6), and $\lambda$ is the regularization parameter controlling the contribution of the two terms.

While the $l_{2}$ norm is used for the data fidelity term (stemming also from a Gaussian assumption for the noise term in a stochastic formulation of the problem), the $l_{1}$ norm is used for the regularization term. Its geometric meaning is explained by 


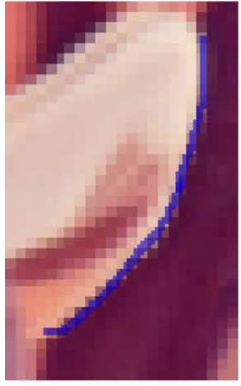

(a)

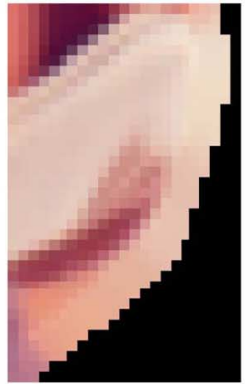

(b)

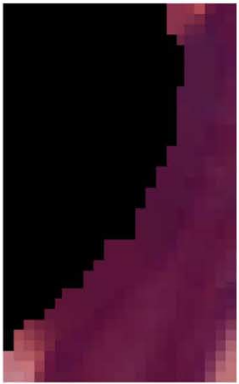

(c)

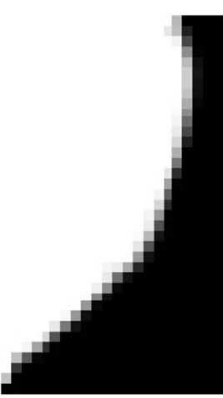

(d)

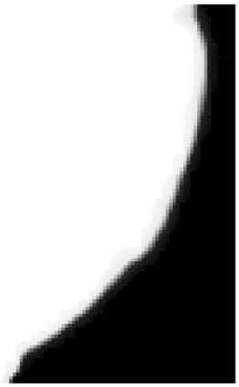

(e)

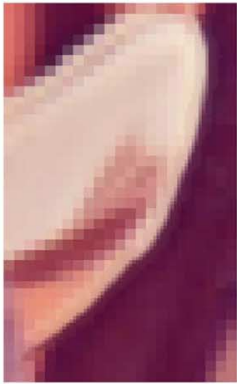

(f)

Fig. 5. Example of $\alpha$-channel edge SR. (a) LR input and the edge needed to be processed. (b), (c) $\mathbf{F}$ and $\mathbf{B}$ on two sides of this edge segment. (d) LR $\alpha$ channel. (e) HR $\alpha$ channel using SoftCuts. (f) Smooth edge generated with matting (8).

Theorem 2 . Besides, the $l_{1}$ norm does not severely penalize large local gradients in general, thus allowing for sharp edges in the restored image (the $l_{2}$ norm results in gradual intensity transition across edges).

The objective function is optimized by the steepest descent algorithm. Denoting by $p_{r e}$ and $p_{s}$ the gradients of the first and second term in (11), we obtain the following iteration:

$$
I^{t+1}=I^{t}-\beta\left(p_{r e}+p_{s}\right)
$$

where

$$
\begin{aligned}
p_{r e}= & \left(\left(I^{t} * G\right) \downarrow-I^{l}\right) \uparrow * G \\
p_{s}= & \lambda \sum_{1 \leq k \leq n_{\mathcal{G}}} w_{k} \\
& \quad \times\left\{\operatorname{sgn}\left(I^{t}-D_{e_{k}} I^{t}\right)\right. \\
& \left.\quad-D_{e_{k}} \operatorname{sgn}\left(I^{t}-D_{e_{k}} I^{t}\right)\right\}
\end{aligned}
$$

$\beta$ is the descent step size and $\uparrow$ denotes the up-sampling operator. $p_{r e}$ is similar to the updating function of the back-projection method [4], except that the back-projection kernel is the same as the blur filter. $D_{e_{k}}$ is the displacement operator, which translates the entire image by $e_{k}$ (an integer valued vector defined in Section III-A), and $\operatorname{sgn}$ is the sign indication function. $p_{s}$ is the derivative of the soft cut metric defined by (6). In fact, each term in (6) will produce a $\left(+w_{k}\right)$ or $\left(-w_{k}\right)$ change for the two corresponding pixels. This updating strategy is the same as in [9]. In our experiments, $I^{0}$ is equal to the bicubic interpolation result.

\section{B. Color Edge SR by Softcuts}

For natural color image SR, a naive solution is to apply the above single channel SR algorithm to all three color channels independently. However, this approach tends to fail for a couple of reasons. First, the SoftCuts method is sensitive to the value of $\lambda$. The selection of this parameter is related to the local edge properties. Some edge strength normalization mechanism is needed to make possible a unified treatment for all edges. Second, in order to determine the exact edge position, information from all three color channels is required. Decisions made on each channel separately might be erroneous and inconsistent.

To address the above issues for color image SR, in this section, we propose to super-resolve color edges by super-resolving its $\alpha$ channel.
Assume that an LR image edge $\mathbf{I}^{l}$ can be decomposed as $\mathbf{I}^{l}=$ $\alpha^{l} \mathbf{F}^{l}+\left(1-\alpha^{l}\right) \mathbf{B}^{l}$. Based on the local smoothness assumption of $\mathbf{F}^{l}$ and $\mathbf{B}^{l}$ mentioned before, it is easy to see that superresolving the edge $\mathbf{I}^{l}$ can be achieved by super-resolving its $\alpha$ channel $\alpha^{l}$. Thus image SR can be performed by processing each image edge separately. To be more specific, for each edge, we first perform alpha matting to a nearby region $\mathbf{I}^{l}$, to obtain $\alpha^{l}, \mathbf{F}^{l}$, and $\mathbf{B}^{l}$. Then their HR counterparts $\alpha^{h}, \mathbf{F}^{h}$, and $\mathbf{B}^{h}$ can be recovered accordingly from them. Recovering $\alpha^{h}$ from $\alpha^{l}=$ $\left(\alpha^{h} * G\right) \downarrow$ is exactly the single channel SR problem discussed in Section V-A, while $\mathbf{F}^{h}$ and $\mathbf{B}^{h}$ can be interpolated using for example the bicubic method, given their down-sampled versions due to the smoothness assumption.

Fig. 5 illustrates the idea of $\alpha$-channel SR for one image edge. The LR patch is decomposed into two image patches and an LR $\alpha$ channel. Fig. 5(e) shows the recovered HR $\alpha$ channel by the proposed SoftCuts method. Combining the images in Fig. 5(b), (c), and (e) using (8) will produce the sharp and smooth edge, shown in Fig. 5(f).

The SoftCuts edge SR method has the following benefits. First, with $\alpha$-channel edge description, each edge is normalized to a unified scale. The same value of $\lambda$ is applied to super-resolve the $\alpha$-channel descriptions of all edges; thus, the problem of parameter selection for the SoftCuts method can be avoided. The underlying rationale is that we want to apply equal weights to all level lines over the $\alpha$ channel of different edges. Besides, the alpha matting technique can extract an edge by combining color information from all three channels; thus, more precise results can be obtained.

However, applying this edge SR method on an entire color image is not easy [28]. It heavily relies on successful extraction of image edges for the LR input image. In addition, to enable edge decomposition by alpha matting, hard constraints for foreground and background pixels need to be automatically specified, which is also quite challenging, and sometimes even impossible.

\section{Adaptive Softcuts for Color Image SR}

In this section, based on the $\alpha$-channel image description, an adaptive method is proposed to overcome the difficulties of applying the $\alpha$-channel SR strategy mentioned above. Based on that, the entire image can be super-resolved in a unified way by implicitly processing all image edges simultaneously. 
We start by considering an image edge, and rewriting (8) as follows:

$$
\alpha=\frac{1}{F^{c}-B^{c}} I^{c}-\frac{B^{c}}{F^{c}-B^{c}}
$$

where $c \in\{r, g, b\}$ is the index for the color channels, and the RGB color space is used in this work. The subscript $p$ in (8) is omitted for simplicity. For a single image edge, the assumption is made that both $\mathbf{F}$ and $\mathbf{B}$ are locally smooth (please refer to Section IV for more details). Thus, (15) shows that the $\alpha$-channel edge description is a linear function of the original image intensity, with scaling factor $1 /\left(F^{c}-B^{c}\right)$, and an approximately constant shift $B^{c} /\left(F^{c}-B^{c}\right)$. This also explains how the edge information is preserved in the $\alpha$-channel, and why the $\alpha$-channel is a normalized description of the image edge. $\left(F^{c}-B^{c}\right)$ in the denominator serves as the normalization factor. More importantly, from (15), we have that the following equation holds locally:

$$
|\alpha|_{\mathcal{G}}=\frac{1}{\left|F^{c}-B^{c}\right|}\left|I^{c}\right|_{\mathcal{G}}
$$

where $|\alpha|_{\mathcal{G}}$ and $\left|I^{c}\right|_{\mathcal{G}}$ are the SoftCuts terms defined by (6) on the $\alpha$-channel and the single color channel, and the constant term is removed due to the smoothness assumption. Equation (16) suggests a locally adaptive strategy for applying the SoftCuts regularization term to the entire image instead of a single edge. More specifically, for different edges, applying the edge adaptation factor $1 /\left|F^{c}-B^{c}\right|$ onto the color channel $c$ can normalize those edges to the $\alpha$-channel, which always has a fixed contrast value of 1 , instead of a large number of possible values in the original color channels. Thus, by applying this adaptation scheme, the same weights are placed on the soft edge smoothness prior for different edges, i.e., the smoothness measures of all the level lines on the $\alpha$ channel are equally weighted.

Now we consider the entire input image with its multiple layer decomposition defined in (9). Based on the above discussion, for each pixel $p$ with two nonzero components in its $\alpha$-channel image description a, the local adaptive factor on the color channel $c$ is

$$
\mu_{p}^{c}=\frac{1}{\left|L_{i, p}^{c}-L_{j, p}^{c}\right|}
$$

where $i$ and $j$ are the layer indices for those two nonzeros components. Since the alpha matting technique does not provide a solution for image layers $\mathbf{L}_{i}$, alternatively, from (16), we use the following equation as an estimator of the above adaptive factor:

$$
\mu_{p}^{c}=\frac{\left|\nabla \mathbf{a}_{p}\right|_{\infty}}{\sqrt{\left|\nabla I_{p}^{c}\right|^{2}+\gamma}}
$$

where $\gamma$ is a small positive number to avoid numerical problems, $|\cdot|_{\infty}$ is the $l_{\infty}$ norm, which returns the largest absolute value of the $\alpha$-channel gradient among all layers. Since most pixels do have one or two nonzero $\alpha$ components [7], the above normalization factor can successfully normalize most part of the image in the same way as in (16), and also gives reasonable results for other parts, as shown in the experiments. Due to the smoothness assumption of image layers, $\mu^{c}$ should also be smooth. To
Input LR image $\mathbf{I}^{l}$ and the scaling factor $s$. Output HR image $\mathbf{I}^{h}$

1) Decompose $\mathbf{I}^{l}$ by using the spectral matting algorithm [7] to get its $\alpha$-channel image description.

2) Compute the LR adaptive factor based on (18) for each pixel in each color channel.

3) Compute the HR adaptive factor by up-sampling with the bicubic interpolation and the scaling factor $s$.

4) For each color channel, optimize the adaptive SoftCuts objective function in (20), to obtain the high resolution result $\mathbf{I}^{h}$.

Fig. 6. Adaptive SoftCuts algorithm for color image SR.



(a)

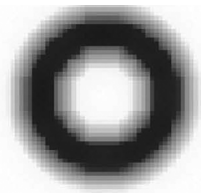

(b)

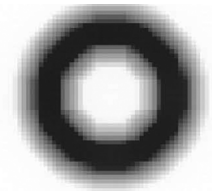

(c)

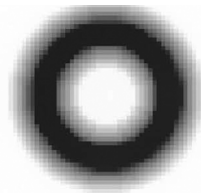

(d)
Fig. 7. (a) LR input image; (b), (c), (d) SR results $(\times 3)$ with soft edge smoothness prior when $n_{\mathcal{G}}=2,4,12$, respectively, $(\lambda=0.01)$.

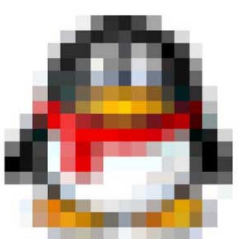

(a)

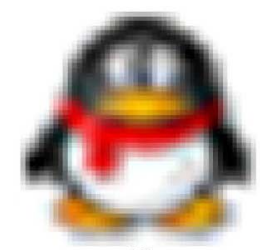

(d)

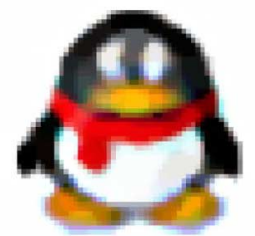

(b)

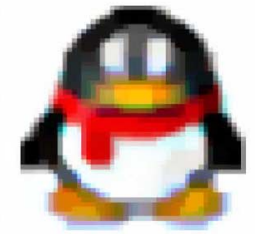

(e)

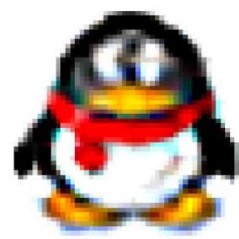

(c)

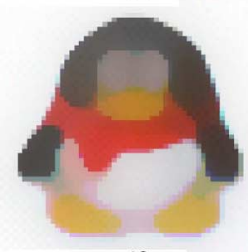

(f)
Fig. 8. Comparison of SR results by soft edge smoothness prior with different parameters $(\times 3)$. (a) LR input image $(20 \times 20)$, (b) $\lambda=0.01, n_{\mathcal{G}}=12$, (c) $\lambda=0.001, n_{\mathcal{G}}=12$, (d) bicubic interpolation, (e) $\lambda=0.01, n_{\mathcal{G}}=2$, (f) $\lambda=0.1, n_{\mathcal{G}}=12$.

suppress image noise, in practice, we convolve the weight map given by (18) with a Gaussian kernel ( $\sigma=1.8$ in our experiments). Please notice that given an LR input image, the resulting alpha description is also in LR. The LR adaptive factor obtained by (18) is then up-sampled with bicubic interpolation to get the HR adaptive factor. Here, bicubic interpolation does not introduce artifacts due to the smoothness property of the adaptive factor. Besides, since different sampling rates do not change the value of the RHS of (18) at the same position, so we do not need to scale the value during up-sampling.

Finally, the adaptive SoftCuts regularization term for color channel $c \in\{r, g, b\}$ is defined as follows:

$$
\left|I^{c}\right|_{\mathcal{G}}^{\prime}=\sum_{(p, q) \in \mathcal{N}}\left(w_{p q} \mu_{p q}^{c}\left|I_{p}^{c}-I_{q}^{c}\right|\right)
$$




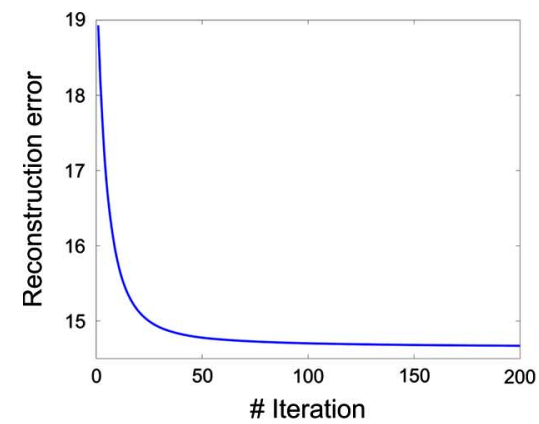

(a) $\left\|I^{I}-(I * G) \downarrow\right\|_{2}^{2}$

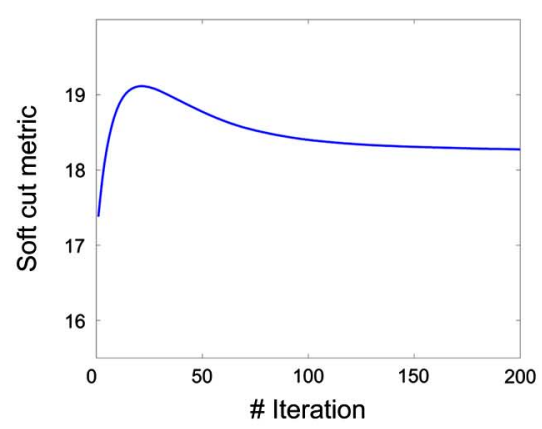

(b) $\lambda|I| g$

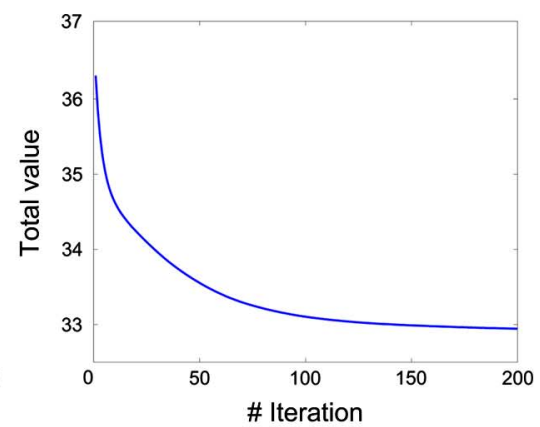

(c) $\|I-(I * G) \downarrow\|_{2}^{2}+\lambda \mid \eta_{g}$

\section{Thanks! Thanks! Thanks! Thanks! Thanks!}

(d)

(e)

(f)

(g)

(h)

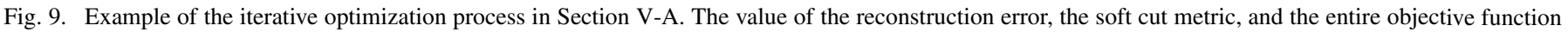

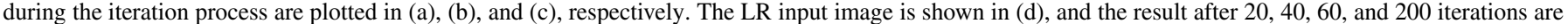
shown in (e), (f), (g), and (h), respectively.

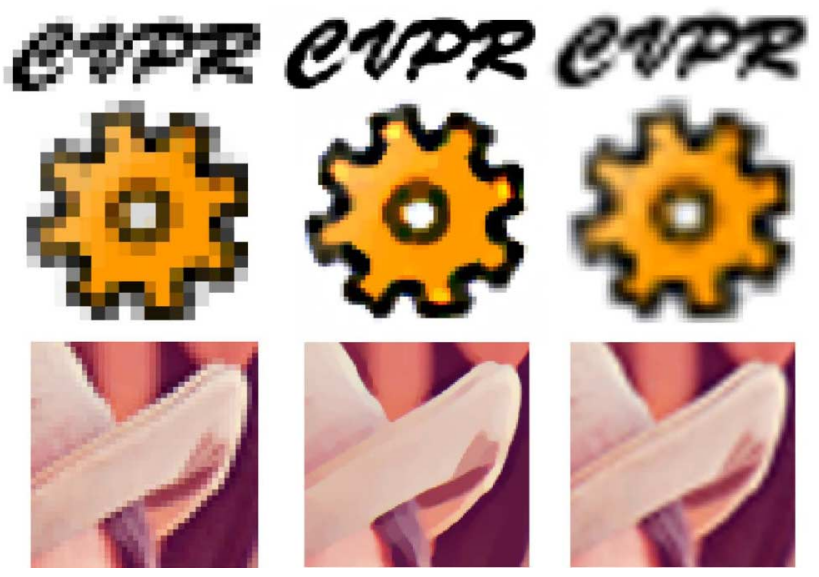

Fig. 10. SR results $(\times 3)$ with soft edge smoothness prior; first column: LR inputs; second column: SR results $\left(\lambda=0.01, n_{\mathcal{G}}=12\right)$; third column: bicubic interpolation.

where $\mu_{p q}^{c}=\left(\mu_{p}^{c}+\mu_{q}^{c}\right) / 2$, and $\mu_{p}^{c}$ and $\mu_{q}^{c}$ are the local adaptive factors defined in (18). The final objective function for adaptive SoftCuts on each channel $c$ is

$$
I^{h, c}=\arg \min _{I^{c}}\left(\left\|I^{l, c}-\left(I^{c} * G\right) \downarrow\right\|_{2}^{2}+\lambda\left|I^{c}\right|_{\mathcal{G}}^{\prime}\right)
$$

Similar iterative method as in Section V-A is applied for optimization.

The entire algorithm is summarized in Fig. 6. Applying the adaptive SoftCuts regularization term to each color channel provides a unified solution by processing all image edges simultaneously. The reliance on the success of the edge extraction algorithms and hard constraint selection for matting are avoided. The benefits, however, of performing SR with the $\alpha$-channel edge description is still materialized.

\section{EXPERIMENTS}

In this section, we first present the SR results of applying SoftCuts on single channel to demonstrate its ability to generate
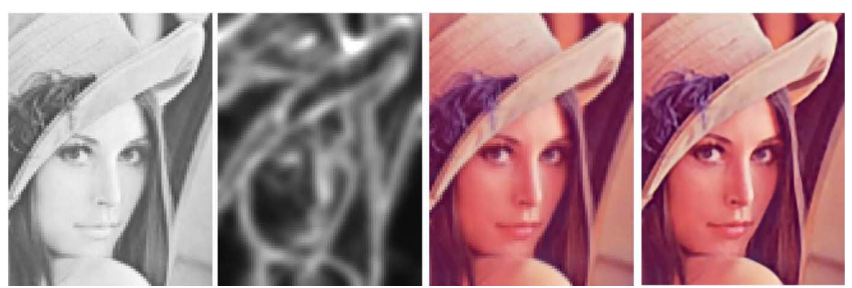

Fig. 11. Visualization of the adaptation weight. From left to right: one LR color channel, LR adaptation weights for this channel (large values of weights are represented by brighter intensity value), color LR input image, and result of the proposed adaptive SoftCuts method.

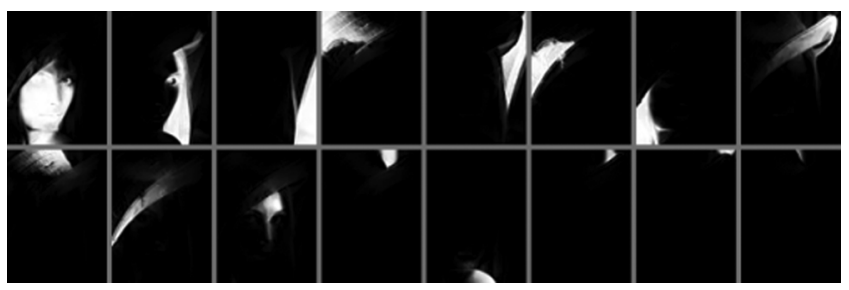

Fig. 12. Matting components extracted by the spectral matting algorithm. The original image is shown in Fig. 11.

smooth edges, and then show the results by using the adaptive SoftCuts method for single color image SR.

\section{A. Single Channel SR by Softcuts}

Fig. 7 shows a proof-of-concept experiment, illustrating the necessity of using higher order neighborhood. Jaggy effects can be observed for small $n_{\mathcal{G}}$, especially in (b) for a 4-neighborhood system. There are some $45^{\circ}$ artifacts in (c), since 8-neighborhood system is used for it. The soft edge is much smoother in (d) with $n_{\mathcal{G}}=12$.

Fig. 8 shows the result comparison of different parameter settings with an LR icon image (icon image SR is also studied in [18]). Larger $n_{\mathcal{G}}$ is applied in (b) than in (e); thus, smoother edges are produced. In (c), a smaller $\lambda$ is used than in (b); thus, a smaller weight is placed on the smoothness prior. This makes the result look over-sharpened on high contrast edges, while 


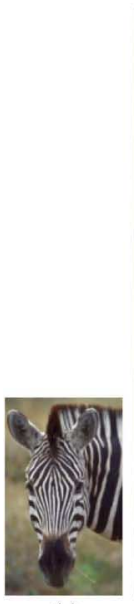

(a)

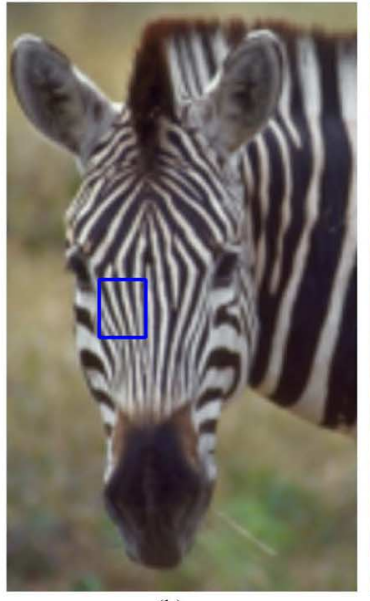

(b)

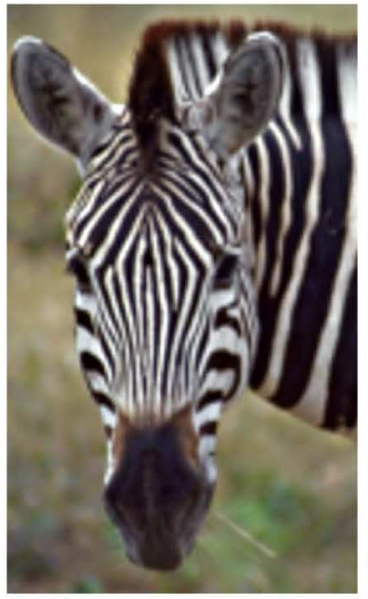

(c)

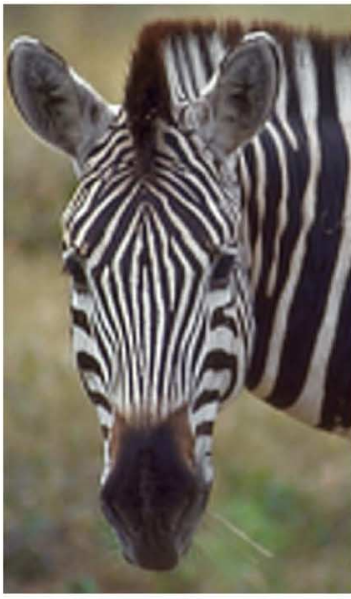

(d)

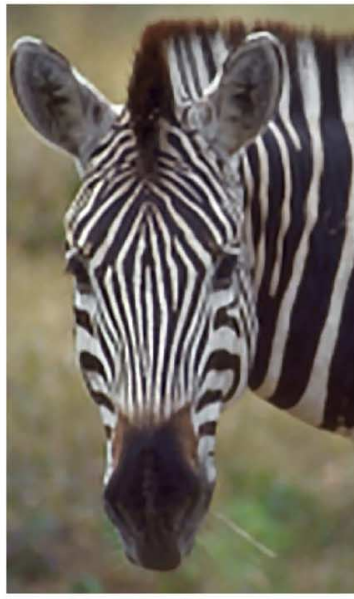

(f)

Fig. 13. Comparison results: (a) LR input, (b) bicubic, (c) bicubic followed by unsharp masking, (d) back-projection [4], (e) proposed method $(\times 3)$.

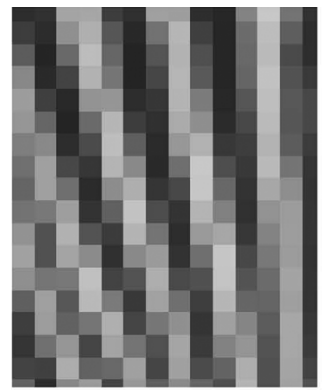

(a)

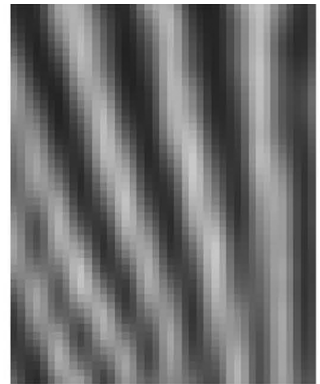

(b)



(c)

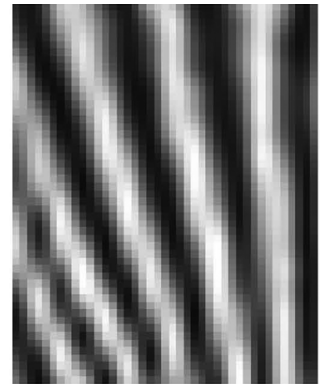

(d)

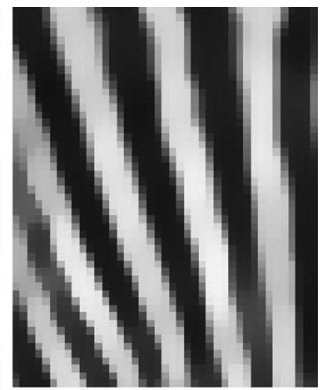

(e)

Fig. 14. Close-up view of the comparison results in Fig. 13(b): (a) LR input, (b) bicubic, (c) bicubic followed by unsharp masking, (d) back-projection [4], (e) proposed method. The selected image patch is highlighted in Fig. 13(b) by a blue box.

a better result is obtained in other parts (such as the foot). In (f), a larger $\lambda$ is used than in (b), the edge smoothness prior is over-weighted. All boundaries are very smooth, but the result is very blurry.

The effect of the parameters can be summarized as follows: (1) a larger $n_{\mathcal{G}}$ will produce smoother boundaries, and is also more computational expensive. In all of the later experiments, $n_{\mathcal{G}}$ is set equal to 12 , with the neighborhood system shown in Fig. 2. (2) The value of $\lambda$ is critical. As revealed by (18), the desirable weight should consider both image gradient and $\alpha$-channel gradient. Besides, the filter $G$ in the generation model (10) also influences the quality of the result. However, estimating $G$ is beyond the scope of this paper. We fix it as a Gaussian filter with $\sigma=1.4$ throughout this paper.

Fig. 9 shows an example of how each term in the objective function [in (11)] changes during the iteration (12). In the first several steps (about 20 in this example), the change of the reconstruction error dominates, and the soft cut metric may increase. After that, although the reconstruction error can hardly be further reduced, the result still looks jaggy as shown in (e). The soft cut metric is further reduced by the iteration, and results in the final result with smooth and sharp edges in (h).

Additional results are shown in Fig. 10. The resulting edges are smooth and with sharp transitions, even when the quality of the LR input images is very low. However, for natural color images like the one in the third row of Fig. 10, although much

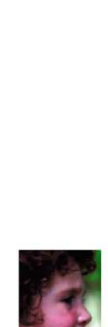

(a)

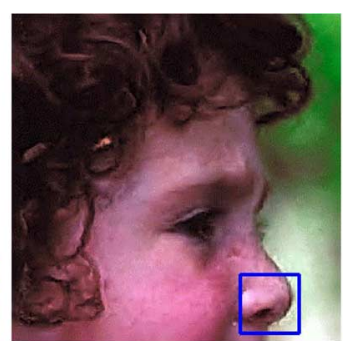

(b)

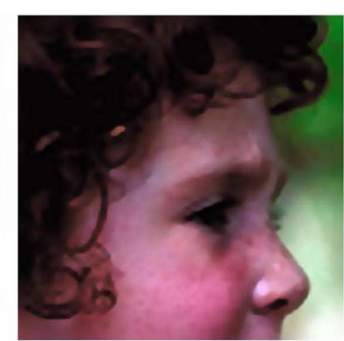

(c)
Fig. 15. Comparison results with exemplar-based methods $(\times 4)$ : (a) LR input, (b) result in [21], (c) result of the proposed method.

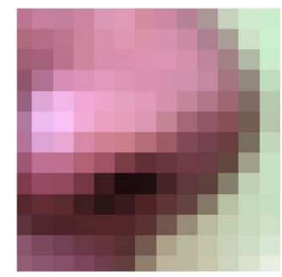

(a)

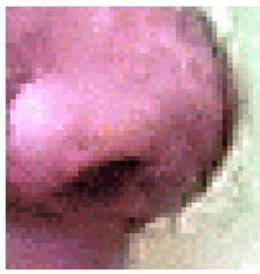

(b)

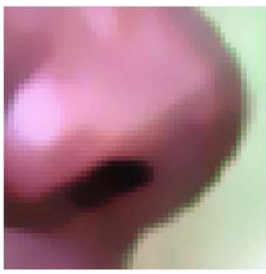

(c)
Fig. 16. Close-up view of the comparison results in Fig. 15: (a) LR input, (b) result in [21], (c) result of the proposed method. The selected image patch is highlighted in Fig. 15(b) by a blue box.

smoother edges are obtained, the image looks unnatural. Some subtle edges are smoothed out. As discussed, the reason is that 


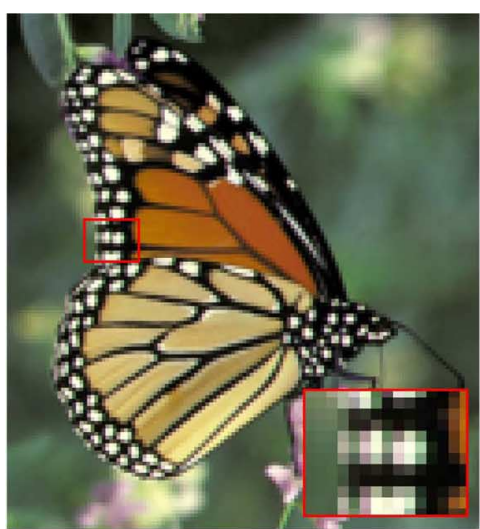

(a) LR input image

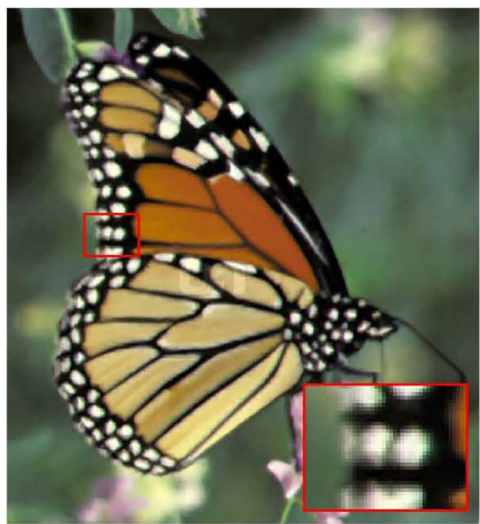

(d) Genuine Fractals 5.0

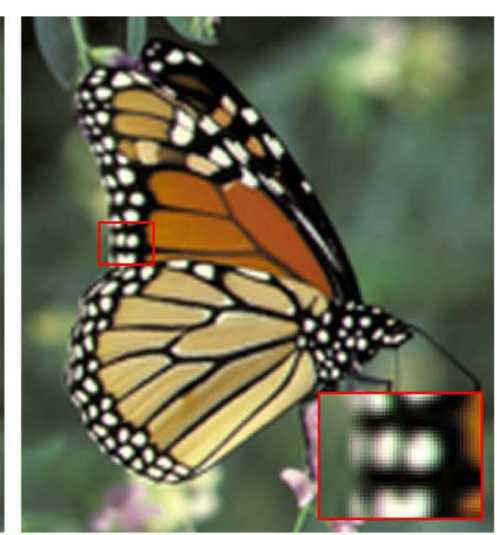

(b) Irfan View Lanczos Interpolation

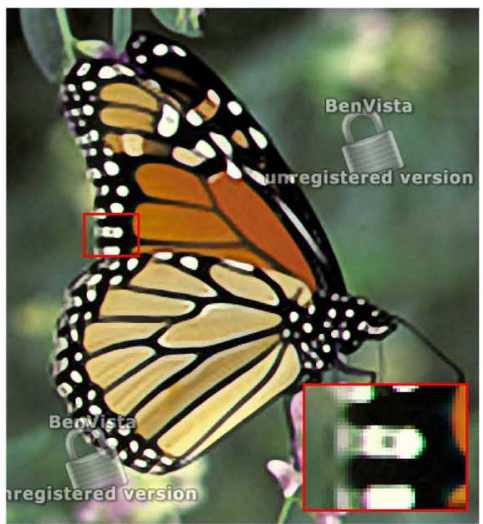

(e) PhotoZoom Pro 2

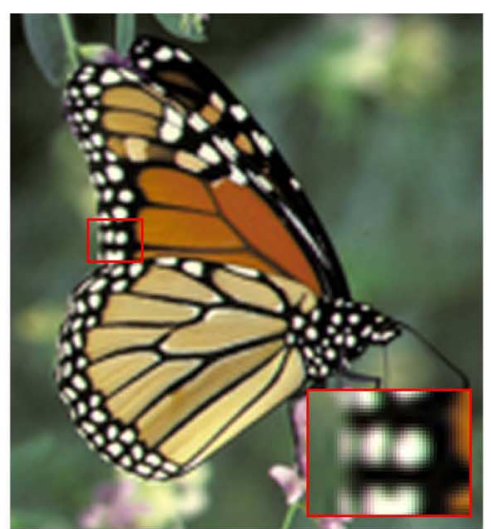

(c) VSO Image Resizer

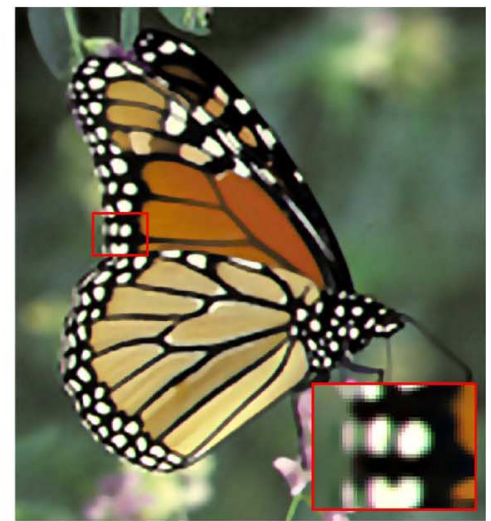

(f) Our result

Fig. 17. Comparison with some existing commercial/free softwares designed specifically for image resizing.

it is difficult, or even impossible, to find a single value of $\lambda$ to address all edges in an image. That is why we need to work on the $\alpha$ channel to normalize different edges, and further develop the proposed adaptive SoftCuts method.

\section{B. Adaptive SoftCuts for Color Image SR}

Fig. 11 displays the adaptation weights for one color channel. Heavier weights are placed on low contrast edges (e.g., those in the regions of the hat and nose), and smaller weights are placed on high contrast edges. Thus, the influence of the smoothness prior on different edges is balanced. Please also notice that a zero weight is assigned to smooth regions; thus, some subtle image fluctuation in those regions could still be present. This makes the result look much more natural than the result with the nonadaptive SoftCuts method shown in Fig. 10. The intermediate result from the spectral matting decomposition is shown in Fig. 12, where the image edge information is clearly extracted.

Fig. 13 shows an example for comparison with other widely used algorithms, including bicubic interpolation, bicubic followed by unsharp masking (Photoshop), and the back-projection method [4]. Fig. 14 shows the close-up views. Blurry edges can be observed with the result of bicubic interpolation. The unsharp masking method (a large radius of 10 , is used to better improve the image contrast) and the back-projection method can increase the image contrast, but the results still look jaggy. Both blurry and jaggy artifacts are successfully removed by the proposed adaptive SoftCuts method, even for very fine image structures.

Fig. 15 compares the proposed method with an exemplarbased algorithm [21]. The existing exemplar-based methods can produce very sharp edges, but rely heavily on effective training data. Compared with it, smoother boundaries can be archived by our method, thus making the result look natural. Fig. 16 shows close-up views to better illustrate the effectiveness of the proposed method. The image boundaries of our result are both smooth and with sharp transitions at the same time.

Fig. 17 compares the proposed method with some existing commercial/free softwares designed specifically for image resizing, including IrfanView Lanczos Interpolator [40], VSO Image Resizer [41], Genuine Fractals 5.0 [42], and PhotoZoom Pro 2 [43]. Close-up views are also shown. Image edges in our result looks smooth, sharp, and natural.

Additional results are shown in Fig. 18. ${ }^{1}$ Various images are tested, and visually appealing results are obtained by the adaptive SoftCuts algorithm. Please notice that the same set of parameters are applied for all of these images, except that the weight of the regularization term $\lambda$ is set equal to 0.01 for natural images, and 0.02 for graphic images. The reason is that the graphic images usually have much smoother edges; thus, a larger weight should be placed on the regularization.

\footnotetext{
${ }^{1}$ More on http://vision.eecs.northwestern.edu/research/IP/SR/index.html.
} 

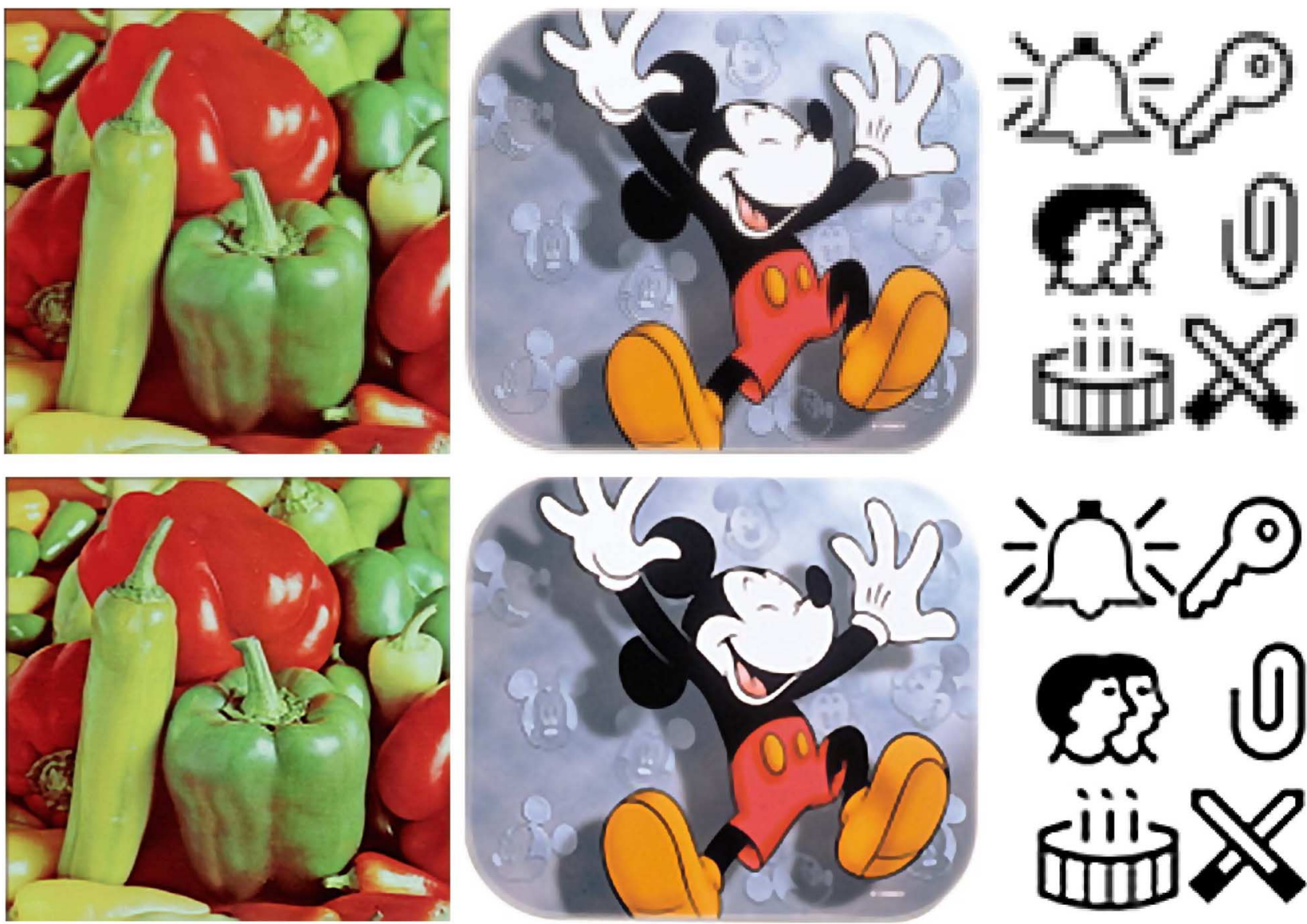

Fig. 18. Additional results with the proposed adaptive SoftCuts method. For each pair of images, the upper one is the LR input, and the lower one is our result $(\times 3)$.

TABLE I

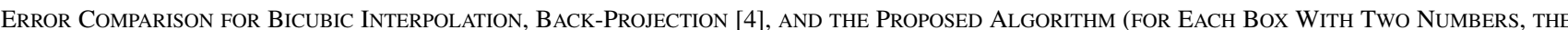
FIRST ONE IS THE RMS ERROR, THE SECOND ONE IS THE ERMS ERROR)

\begin{tabular}{|c|c|c|c|c|c|c|c|}
\hline & Lena & Boy & Zebra & Monarch & Peppers & Fonts & Mickey \\
\hline Bicubic & $\begin{array}{lll}9.14 & 17.99\end{array}$ & $10.12 \quad 14.92$ & 9.5929 .82 & 9.4332 .60 & 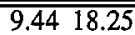 & 12.4766 .85 & 99.7429 .89 \\
\hline BP [4] & $\begin{array}{lll}7.52 & 11.87\end{array}$ & $9.36 \quad 12.99$ & 7.9919 .48 & $7.65 \quad 17.93$ & 8.6912 .95 & 11.1053 .78 & 9.8727 .08 \\
\hline Ours & $\begin{array}{lll}7.88 & 12.73\end{array}$ & 9.5413 .41 & $\begin{array}{lll}7.62 & 19.97\end{array}$ & 7.6418 .53 & $\begin{array}{lll}8.70 & 12.88\end{array}$ & 8.7145 .50 & $9.28 \quad 26.30$ \\
\hline
\end{tabular}

Quantitatively, the RMS errors (root-of-mean-square error) for bicubic interpolation, back-projection, and the proposed SoftCuts method is shown in Table I. The back-projection method [4] is effective in reducing the RMS error by enforcing the reconstruction constraint. The RMS error for our algorithm is roughly the same as the back-projection method, since a similar reconstruction constraint is enforced. However, the back-projection algorithm aims at minimizing only the data fidelity term, which is reflected in the RMS error. Therefore, in certain cases, the back-projection algorithm results in a smaller RMS error, although the visual quality of the results is not as good as the one provided by the proposed algorithm. Due to the additional soft edge smoothness prior, some improvement is observed for images with smooth and sharp edges, such as Zebra, Fonts, and Mickey. The ERMS (edge RMS) errors are also presented, since image edges are more important for visual perception. Similar observation can be made with the ERMS error.
For complexity, we run our experiments on a PIV3.4G PC with 1-G RAM by Matlab implementation. For an LR input image of size $107 \times 160$ pixels, the spectral matting algorithm for image decomposition takes $120 \mathrm{~s}$, and the adaptive SoftCuts method takes $35 \mathrm{~s}$ for 30 iterations.

\section{CONCLUSION}

In this paper, a novel single image SR algorithm is proposed. A soft edge smoothness measure is defined on a large neighborhood system, which is an approximation of the average length of all level lines in the image. To extend this method to natural color image SR, an adaptive SoftCuts method is proposed based on a novel $\alpha$-channel image description. It enables a unified treatment of edges with different contrasts on the $\alpha$ channel. Promising results for a large variety of images are obtained by this algorithm. 


\section{APPENDIX \\ PROOF OF THEOREM 2}

From (5), we have

$$
\left|l_{i}\right|_{\mathcal{G}}=\sum_{k}\left(w_{k} \sum_{\mathcal{N}_{k}}\left|\chi_{p}^{l_{i}}-\chi_{q}^{l_{i}}\right|\right)
$$

thus

$$
\begin{aligned}
\sum_{i}\left|l_{i}\right|_{\mathcal{G}} & =\sum_{i} \sum_{k}\left(w_{k} \sum_{\mathcal{N}_{k}}\left|\chi_{p}^{l_{i}}-\chi_{q}^{l_{i}}\right|\right) \\
& =\sum_{k}\left(w_{k} \sum_{\mathcal{N}_{k}} \sum_{i}\left|\chi_{p}^{l_{i}}-\chi_{q}^{l_{i}}\right|\right) \\
& \text { and } \\
\sum_{i}\left|\chi_{p}^{l_{i}}-\chi_{q}^{l_{i}}\right| & =\#\left\{i \mid \chi_{p}^{l_{i}} \neq \chi_{q}^{l_{i}}\right\} \\
& =n \cdot\left|S_{p}^{d}-S_{q}^{d}\right|
\end{aligned}
$$

resulting in

$$
\begin{aligned}
\sum_{i}\left|l_{i}\right|_{\mathcal{G}} & =\sum_{k}\left(w_{k} \sum_{\mathcal{N}_{k}} n \cdot\left|S_{p}^{d}-S_{q}^{d}\right|\right) \\
& =n \cdot \sum_{k}\left(w_{k} \sum_{\mathcal{N}_{k}}\left|S_{p}^{d}-S_{q}^{d}\right|\right) \\
& =n \cdot\left|S^{d}\right|_{\mathcal{G}} \quad(\text { from Eqn. } 6) .
\end{aligned}
$$

Thus, from Theorem 1, we have

$$
\left|S^{d}\right|_{\mathcal{G}}=\frac{1}{n} \sum_{i}\left|l_{i}\right|_{\mathcal{G}} \rightarrow \frac{1}{n} \sum_{i}\left|l_{i}\right|_{\mathcal{E}}
$$

\section{REFERENCES}

[1] Y. Boykov and V. Kolmogorov, "Computing geodesics and minimal surfaces via graph cuts," presented at the ICCV, 2003.

[2] A. Katsaggelos, R. Molina, and J. Mateos, Super Resolution of Images and Video, Synthesis Lectures on Image, Video, and Multimedia Processing. New York: Morgan \& Claypool, 2007.

[3] S. Chaudhuri and J. Manjunath, Motion-Free Super-Resolution. New York: Springer, 2005.

[4] M. Irani and S. Peleg, "Motion analysis for image enhancement: Resolution, occlusion and transparency," JVCIP, 1993.

[5] S. Baker and T. Kanade, "Limits on super-resolution and how to break them," IEEE Trans. Pattern Anal. Mach. Intell., vol. 24, no. 9, pp. 1167-1183, Sep. 2002.

[6] Z. Lin and H.-Y. Shum, "Fundamental limits of reconstruction based super-resolution algorithms under local translation," IEEE Trans. Pattern Anal. Mach. Intell., vol. 26, no. 1, pp. 83-97, Jan. 2004.

[7] A. Levin, A. Rav-Acha, and D. Lischinski, "Spectral matting," presented at the CVPR, 2007.

[8] M. Elad and A. Feuer, "Restoration of single super-resolution image from several blurred, noisy and down-sampled measured images," IEEE Trans. Image Process., vol. 6, no. 12, pp. 1646-1658, Dec. 1997.

[9] S. Farsiu, M. D. Robinson, M. Elad, and P. Milanfar, "Fast and robust multiframe super resolution," IEEE Trans. Image Process., vol. 13, no. 10, pp. 1327-1344, Oct. 2004.

[10] M. F. Tappen, B. Russell, and W. T. Freeman, "Exploiting the sparse derivative prior for super-resolution and image demosaicing," presented at the IEEE Workshop on Statistical and Computational Theories of Vision, 2003.

[11] S. D. Babacan, R. Molina, and A. K. Katsaggelos, "Total variation super resolution using a variational approach," presented at the Int. Conf. Image Processing, 2008.

[12] S. Farsiu, M. Elad, and P. Milanfar, "Multi-frame demosaicing and super-resolution of color images," IEEE Trans. Image Process., vol. 15, no. 1, pp. 141-159, Jan. 2006.

[13] J. Allebach and P. W. Wong, "Edge-directed interpolation," presented at the Int. Conf. Image Processing, 1996.

[14] X. Li and M. Orchard, "New edge-directed interpolation," IEEE Trans. Image Process., vol. 10, no. 10, pp. 1521-1527, Oct. 2001.
[15] D. D. Muresan, "Fast edge directed polynomial interpolation," presented at the Int. Conf. Image Processing, 2005.

[16] B. S. Morse and D. Schwartzwald, "Image magnification using level set reconstruction," presented at the CVPR, 2001.

[17] Y.-W. Tai, W.-S. Tong, and C.-K. Tang, "Perceptually-inspired and edge-directed color image super-resolution," presented at the CVPR, 2006.

[18] V. Rabaud and S. Belongie, "Big little icons," CVAVI, 2005.

[19] R. Nakagaki and A. K. Katsaggelos, "A vq-based blind image restoration algorithm," IEEE Trans. Image Process., vol. 12, no. 9, pp. 1044-1053, Sep. 2003.

[20] W. T. Freeman, T. R. Jones, and E. C. Pasztor, "Example-based superresolution," IEEE Comput. Graph. Appl., 2002.

[21] W. T. Freeman, E. Pasztor, and O. Carmichael, "Learning low-level vision," Int. J. Comput. Vis., vol. 40, no. 1, pp. 25-47, 2000.

[22] C. Liu, H.-Y. Shum, and C.-S. Zhang, "A two-step approach to hallucinating faces: Global parametric model and local nonparametric model," presented at the CVPR, 2001.

[23] C. M. Bishop, A. Blake, and B. Marthi, "Super-resolution enhancement of video," presented at the Artificial Intelligence and Statistics, 2003.

[24] D. Kong, M. Han, W. Xu, H. Tao, and Y. Gong, "Video super-resolution with scene-specific priors," presented at the BMVC, 2006.

[25] Q. Wang, X. Tang, and H. Shum, "Patch based blind image super resolution," presented at the CVPR, 2005.

[26] J. Sun, N. Zheng, H. Tao, and H. Shum, "Image hallucination with primal sketch priors," presented at the CVPR, 2003.

[27] H. Chang, D. Yeung, and Y. Xiong, "Super-resolution through neighbor embedding," presented at the CVPR, 2004.

[28] S. Dai, M. Han, W. Xu, Y. Wu, and Y. Gong, "Soft edge smoothness prior for alpha channel super resolution," presented at the CVPR, 2007.

[29] Y. Boykov and V. Kolmogorov, "An experimental comparison of min-cut/max-flow algorithms for energy minimization in vision," IEEE Trans. Pattern Anal. Mach. Intell., vol. 26, no. 9, pp. 1124-1137, Sep. 2004.

[30] Y. Boykov, O. Veksler, and R. Zabih, "Fast approximate energy minimization via graph cuts," IEEE Trans. Pattern Anal. Mach. Intell., vol. 23, no. 11, pp. 1222-1239, Nov. 2001.

[31] L. Rudin, S. Osher, and E. Fatemi, "Nonlinear total variation based noise removal algorithms," Phys. D, vol. 60, pp. 259-268, 1992.

[32] T. F. Chan, S. Osher, and J. Shen, "The digital tv filter and nonlinear denoising," IEEE Trans. Image Process., vol. 10, no. 2, pp. 231-241, Feb. 2001.

[33] S. Babacan, R. Molina, and A. Katsaggelos, "Parameter estimation in tv image restoration using variational distribution approximation," IEEE Trans. Image Process., vol. 17, no. 3, pp. 326-339, Mar. 2008.

[34] A. Levin, D. Lischinski, and Y. Weiss, "A closed form solution to natural image matting," IEEE Trans. Pattern Anal. Mach. Intell., 2007.

[35] J. Jia, "Single image motion deblurring using transparency," presented at the CVPR, 2007.

[36] Q. Shan, W. Xiong, and J. Jia, "Rotational motion deblurring of a rigid object from a single image," presented at the ICCV, 2007.

[37] S. Dai and Y. Wu, "Motion from blur," presented at the CVPR, 2008.

[38] E. P. Bennett, M. Uyttendaele, C. L. Zitnick, R. Szeliski, and S. B. Kang, "Video and image bayesian demosaicing with a two color image prior," ECCV, 2006.

[39] J. Wang, M. Agrawala, and M. F. Cohen, "Soft scissors: An interactive tool for realtime high quality matting," ACM Trans. Graphics (Proc. SIGGRAPH), 2007.

[40] Irfanview [Online]. Available: http://www.irfanview.com/

[41] VSO Image Resizer [Online]. Available: http://www.vso-software.fr

[42] Genuine Fractals 5.0 [Online]. Available: http://www.ononesoftware.com

[43] Photozoom Pro 2 [Online]. Available: http://www.benvista.com

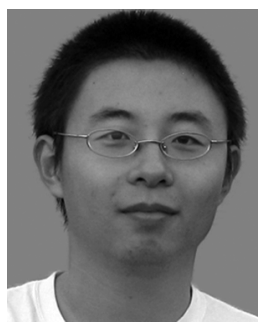

Shengyang Dai (S'05) received the B.S. and M.S. degrees from the Electrical Engineering Department, Tsinghua Univresity, Beijing, China, in 2001 and 2004 , respectively. He is currently pursuing the $\mathrm{Ph} . D$. degree in the Electrical Engineering and Computer Science Department, Northwestern University, Evanston, IL.

His research interests include image/video processing, computer vision, and machine learning. $\mathrm{He}$ did summer internships at NEC Laboratories America (Cupertino, CA), Microsoft Research (Redmond, WA), and Google Research (Mountain View, CA) in 2006, 2007, and 2008, respectively.

Mr. Dai received the Outstanding Graduate Student Fellowship from Tsinghua University in 2004 and the Everly Fellowship at Northwestern University in 2008 . 


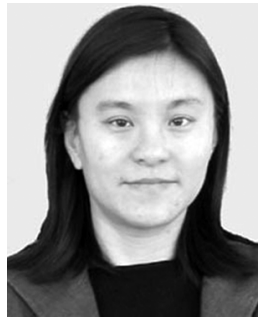

Mei Han received the B.S. and Ph.D. degrees in computer science from Tsinghua University, China, in 1992 and 1995, and the Ph.D. degree in Robotics from Carnegie Mellon University, Pittsburgh, PA, in 2001.

From 2001 to 2007, she was a research staff member at NEC Laboratories America, Cupertino, CA. She has been a research scientist at Google, Inc. since August 2007. Her research interests include computer vision, computer graphics, image and video analysis, video surveillance, multimedia data mining, and machine learning.

Dr. Han served as a committee member for the ECCV 2002 Workshop on Vision and Modeling of Dynamic Scenes and a Session Chair for the International Conference on Image Processing 2004.

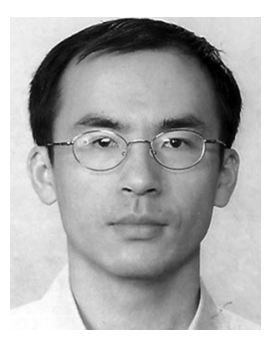

Wei Xu received the B.S. degree from Tsinghua University, Beijing, China, in 1998, and the M.S. degree from Carnegie Mellon University (CMU), Pittsburgh, $\mathrm{PA}$, in 2000 .

From 1998 to 2001, he was a research assistant at the Language Technology Institute, CMU. In 2001, he joined NEC Laboratories America, Cupertino, $\mathrm{CA}$, working on intelligent video analysis. His research interests include computer vision, image and video understanding, machine learning, and data mining.

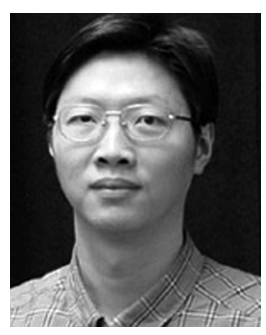

Ying Wu (SM'06) received the B.S. degree from the Huazhong University of Science and Technology, Wuhan, China, in 1994, the M.S. degree from Tsinghua University, Beijing, China, in 1997, and the Ph.D. degree in electrical and computer engineering from the University of Illinois at Urbana-Champaign (UIUC), Urbana, in 2001.

From 1997 to 2001, he was a research assistant at the Beckman Institute for Advanced Science and Technology, UIUC. During the summers of 1999 and 2000, he was a research intern with Microsoft Research, Redmond, WA. In 2001, he joined the Department of Electrical and Computer Engineering, Northwestern University, Evanston, IL, as an Assistant Professor. He is currently an Associate Professor of electrical engineering and computer science at Northwestern University. His current research interests include computer vision, image and video analysis, pattern recognition, machine learning, multimedia data mining, and human-computer interaction.

Dr. Wu serves as an Associate Editor for the IEEE TRANSACTIONS ON IMAGE PROCESSING, the SPIE Journal of Electronic Imaging, and the IAPR Journal of Machine Vision and Applications. He received the Robert T. Chien Award at UIUC in 2001 and the NSF CAREER award in 2003.

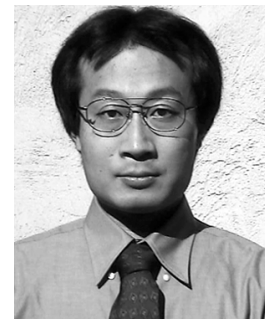

Yihong Gong received the B.S., M.S., and Ph.D. degrees in electronic engineering from the University of Tokyo, Tokyo, Japan, in 1987, 1989, and 1992, respectively.

He joined the Nanyang Technological University (NTU), Singapore, where he was an Assistant Professor at the School of Electrical and Electronic Engineering. Between June 1996 and December 1998, he was with the Robotics Institute, Carnegie Mellon University, Pittsburgh, PA, as a project scientist and principal member of both the Informedia Digital Video Library project and the Experience-On-Demand project funded by NSF, DARPA, and other governmental agencies. In 1999, he joined NEC Laboratories America, Cupertino, CA, where he built the multimedia content analysis team from scratch. In 2006, he became Head of the silicon valley branch of the labs. His research interests include computer vision, multimedia content analysis, and machine learning.

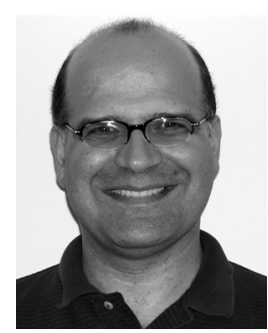

Aggelos K. Katsaggelos (S'80-M'85-SM'92-F'98) received the Diploma degree in electrical and mechanical engineering from the Aristotelian University of Thessaloniki, Thessaloniki, Greece, in 1979, and the M.S. and Ph.D. degrees in electrical engineering from the Georgia Institute of Technology, Atlanta, in 1981 and 1985, respectively.

In 1985, he joined the Department of Electrical and Computer Engineering, Northwestern University, Evanston, IL, where he is currently a Professor He held the Ameritech Chair of Information Technology from 1997 to 2003. He is also the Director of the Motorola Center for Communications and a member of the Academic Affiliate Staff, Department of Medicine, Evanston Hospital. He is the editor of Digital Image Restoration (Springer-Verlag, 1991), coauthor of Rate-Distortion Based Video Compression (Kluwer, 1997), coeditor of Recovery Techniques for Image and Video Compression and Transmission (Kluwer, 1998), and co author of Super-Resolution for Images and Video (Claypool, 2007) and Joint Source-Channel Video Transmission (Claypool, 2007), as well as the coinventor of 12 patents.

Dr. Katsaggelos has served the IEEE and other professional societies in many capacities. For example, he was Editor-in-Chief of the IEEE Signal Processing Magazine (1997-2002), a member of the Board of Governors of the IEEE Signal Processing Society (1999-2001), and a member of the Publication Board of the IEEE PROCEEDINGS (2003-2007). He is the recipient of the IEEE Third Millennium Medal (2000), the IEEE Signal Processing Society Meritorious Service Award (2001), an IEEE Signal Processing Society Best Paper Award (2001), an IEEE International Conference on Multimedia and Expo Paper Award (2006), and an IEEE International Conference on Image Processing Paper Award (2007). He is a Distinguished Lecturer of the IEEE Signal Processing Society (2007-2008). 